\title{
Causas de readmissão hospitalar após cirurgia cardíaca
}

\author{
Bárbara Rodrigues Nunes Barreiros ${ }^{1}$, Estela Regina Ferraz Bianchi ${ }^{2}$, Ruth Natalia Teresa Turrini ${ }^{3}$, \\ Vanessa de Brito Poveda ${ }^{4}$
}

${ }^{1}$ Enfermeira. São Paulo, SP, Brasil. E-mail: bahbarreiros@hotmail.com.

${ }^{2}$ Enfermeira, Doutora em Enfermagem. Assessora de Pesquisa em Enfermagem do Instituto Dante Pazzanese de Cardiologia. São Paulo, SP, Brasil. E-mail: estelarfb@gmail.com.

${ }^{3}$ Enfermeira, Doutorado em Saúde Pública. Professor Livre Docente da da Escola de Enfermagem da Universidade de São Paulo. São Paulo, SP, Brasil. E-mail: rturrini@usp.br.

${ }^{4}$ Enfermeira, Doutora em Enfermagem. Professora Doutor da Escola de Enfermagem da Universidade de São Paulo. São Paulo, SP, Brasil. E-mail: vbpoveda@usp.br.

Recebido: 26/01/2016.

Aceito: 11/07/2016.

Publicado: 08/12/2016.

Como citar esse artigo: Barreiros BRN, Bianchi ERF, Turrini RNT, Poveda VB. Causas de readmissão hospitalar após cirurgia cardíaca. Rev. Eletr. Enf. [Internet]. 2016 [acesso em: ___;18:e1182. Disponível em: http://dx.doi.org/10.5216/ree.v18.39529.

\section{RESUMO}

Objetivou-se identificar o perfil e as causas de readmissão de pacientes submetidos à cirurgia cardíaca. Estudo retrospectivo, descritivo, realizado por meio de revisão de prontuários de pacientes submetidos à cirurgia de revascularização do miocárdio ou implante de prótese valvar com readmissão posterior. A amostra foi constituída por 62 pacientes. A taxa de readmissão foi de 5,9\%. A infecção do sítio cirúrgico foi a principal causa de readmissão em $87,5 \%$ dos pacientes submetidos à revascularização do miocárdio e em $12,5 \%$ dos implantes de prótese valvar $(p<0,001)$ e esteve associada às variáveis obesidade $(p=0,05)$ e dislipidemia $(p=0,007)$. A identificação dos pacientes em risco para o desenvolvimento de infecção do sítio cirúrgico pode minimizar as taxas de readmissão e diminuir os custos associados ao cuidado, e, merece um planejamento diferenciado de ações multiprofissionais.

Descritores: Enfermagem Perioperatória; Readmissão do Paciente; Infecção da Ferida Operatória; Procedimentos Cirúrgicos Cardiovasculares.

\section{INTRODUÇÃO}

As doenças crônicas não transmissíveis são um problema de saúde global, destacando-se as cardiovasculares $^{(1)}$, responsáveis por cerca de $20 \%$ das mortes em indivíduos acima de 30 anos. Entre elas, as síndromes coronarianas, representam $80 \%$ destas enfermidades e podem culminar com a necessidade de intervenção cirúrgica ${ }^{(2-3)}$.

Existe ainda, o crescente aumento da realização de cirurgias cardíacas em indivíduos com mais de 65 anos, vinculado ao incremento da expectativa de vida da população, tornando os idosos fortes candidatos 
ao procedimento de revascularização do miocárdio, devido ao agravamento da doença aterosclerótica coronariana $^{(1-5)}$.

O tratamento cirúrgico busca a melhora da qualidade de vida dos pacientes, sobressaindo-se as cirurgias de revascularização do miocárdio (RM) realizada quando ocorre oclusão das artérias coronárias do coração $^{(4)}$ e o implante de prótese valvar (IPV), frequente entre homens com mais de 40 anos e mulheres pós-menopausa(5).

As cirurgias cardíacas elevam a morbidade, relacionada ao estado de saúde, hábitos prévios dos pacientes e complexidade intrínseca do procedimento, que contribuem para que os indivíduos em seu pósoperatório estejam hemodinamicamente instáveis, apresentando complicações frequentes, que aumentam os períodos de internação ou exigem readmissões ${ }^{(6)}$.

Estudo analisando 3.004 hospitais americanos e 479.471 altas hospitalares após procedimentos cirúrgicos de grande porte, entre eles a RM, observou índices de readmissão, em 30 dias, que variavam entre 10,5 e 17,4\%, e acometiam, especialmente, idosos, com comorbidades prévias. Além disso, destaca-se também, a relação entre readmissão e qualidade do cuidado perioperatório oferecido ${ }^{(7)}$.

As taxas de readmissão entre pacientes submetidos a cirurgias cardíacas podem variar entre 6,1 e 18\% e estão, principalmente, associadas à falência cardíaca e infecção ${ }^{(6,8)}$. Como fatores de risco para a ocorrência deste evento destacam-se a idade, comorbidades pré-operatórias, índice de massa corporal, diabetes mellitus, entre outros ${ }^{(9)}$.

Portanto, as readmissões são um problema frequente nas instituições de saúde, o que propicia desconforto ao paciente e seus familiares, onerando a sociedade, ao sobrecarregar o sistema de saúde ${ }^{(10)}$.

Assim, conhecer os motivos das readmissões em um serviço de cardiologia pode favorecer o planejamento da assistência hospitalar, reduzindo-as. Dessa forma, o presente artigo teve como objetivo identificar o perfil e as causas de readmissão dos pacientes submetidos à cirurgia cardíaca.

\section{MÉTODO}

Estudo retrospectivo, descritivo, realizado por meio da revisão de prontuários de pacientes submetidos a cirurgias de RM ou IPV, que sofreram readmissões, em um hospital público de alta complexidade, referência em cardiologia em uma capital do sudeste brasileiro.

A coleta de dados foi realizada de janeiro a abril de 2014, no Serviço de Arquivo Médico e Estatística (SAME), em duas etapas. Em um primeiro momento foram averiguadas as readmissões hospitalares ocorridas em 2012, após os procedimentos cirúrgicos de RM ou IPV, por meio do banco de dados do SAME, sendo coletados: data da internação, unidade, causa, intervalo de tempo entre as internações e tempo que o paciente ficou internado.

Identificaram-se 573 procedimentos de revascularização do miocárdio e 484 substituições de válvula, totalizando 1.057 procedimentos no ano de 2012, dos quais 62 pacientes haviam sofrido readmissão após a cirurgia. 
Em um segundo momento, dos 62 prontuários selecionados foram coletados o perfil sociodemográfico, incluindo sexo, idade, local da residência, estado civil, escolaridade e situação trabalhista. Para a caracterização do atendimento hospitalar foram utilizados os diagnósticos médicos, tratamento recebido, tempo de internação, número de internações e comorbidades como, hipertensão arterial, dislipidemia, tabagismo, obesidade, estresse, sedentarismo e outros. Para caracterizar a readmissão foram analisados a causa, o tempo decorrido entre a readmissão e a internação anterior, diagnóstico médico e a evolução clínica dos pacientes.

Os dados foram submetidos à análise estatística descritiva com o programa SPSS 20.0, utilizando o teste exato de Fisher (variáveis categóricas) e teste $t$ Student (variáveis numéricas) e regressão logística. Foram considerados significativos os valores de $p$ menores ou iguais a 0,05.

Este projeto de pesquisa seguiu as recomendações da resolução 466/2012(11) e foi aprovado pelo Comitê de Ética em Pesquisa do hospital selecionado para estudo sob no de protocolo 4351 (CAAE 18774113.0.0000.5462).

\section{RESULTADOS}

Compuseram a amostra 62 prontuários $(5,9 \%)$ de pacientes que sofreram readmissões no período investigado, representando uma taxa de 6,3\% entre as RM e de 5,4\% entre os IPV.

Dentre as readmissões, 36 (58\%) pacientes haviam sido submetidos à RM e 26 (42\%) à IPV.

A principal causa da primeira readmissão foi a infecção do sítio cirúrgico (ISC) em 24 (38,7\%) pacientes. Apenas nove $(14,5 \%)$ sujeitos foram readmitidos pela segunda vez e um $(1,6 \%)$ pela terceira vez. Nas duas últimas situações, as internações foram motivadas, sobretudo pelo tratamento de complicações como tamponamento cardíaco, derrame pleural, entre outros (Tabela 1).

Tabela 1: Distribuição dos motivos de readmissão de pacientes submetidos à revascularização do miocárdio e implante de prótese valvar. São Paulo, SP, Brasil, 2012.

\begin{tabular}{|c|c|c|c|}
\hline Readmissão & Motivo de readmissão & $\mathbf{N}$ & $\%$ \\
\hline \multirow{7}{*}{$1 \underline{a}$ readmissão } & Infecção do sítio cirúrgico & 24 & 38,7 \\
\hline & $\begin{array}{c}\text { Procedimento clínico/cirúrgico/diagnóstico (cateterismo cardíaco, avaliação marcapasso, novas } \\
\text { cirurgias) }\end{array}$ & 14 & 22,6 \\
\hline & $\begin{array}{c}\text { Problemas cardiovasculares (insuficiência arterial c/ isquemia, insuficiência cardíaca, síndrome } \\
\text { coronariana aguda, miocardiopatia) }\end{array}$ & 9 & 14,5 \\
\hline & $\begin{array}{c}\text { Complicação de procedimento clínico ou cirúrgico (tamponamento cardíaco, derrame pleural, } \\
\text { entre outros) }\end{array}$ & 4 & 6,4 \\
\hline & Complicação neurológica & 3 & 4,8 \\
\hline & Outros (problemas respiratórios, doença reumática, entre outros) & 8 & 12,9 \\
\hline & Total & 62 & 100 \\
\hline \multirow{4}{*}{$2 \underline{a}$ readmissão } & $\begin{array}{c}\text { Complicação de procedimento clínico ou cirúrgico (tamponamento cardíaco, derrame pleural, } \\
\text { entre outros) }\end{array}$ & 3 & 33,3 \\
\hline & Insuficiência arterial com isquemia & 2 & 22,2 \\
\hline & Outros (confecção de fistula arteriovenosa, hemotórax, exames, entre outros) & 4 & 44,4 \\
\hline & Total & 9 & 100 \\
\hline \multirow[t]{2}{*}{ 3a readmissão } & Insuficiência Cardíaca & 1 & 100 \\
\hline & Total & 1 & 100 \\
\hline
\end{tabular}


$\mathrm{Na}$ internação referente à realização do procedimento cirúrgico proposto, 69\% da amostra permaneceu mais de sete dias hospitalizada, $18 \%$ até sete dias e $13 \%$ mais de 30 dias. Os pacientes estiveram fora da instituição hospitalar entre o procedimento cirúrgico proposto e a primeira readmissão, em média, por $51,6 \pm 70,4$ dias, sendo que a duração da estadia hospitalar na primeira readmissão foi, em média, de $16,9 \pm 14,5$ dias (Tabela 2).

Tabela 2: Número de dias por internação, readmissão e período entre as readmissões de pacientes submetidos à revascularização do miocárdio e implante de prótese valvar. São Paulo, SP, Brasil, 2012.

\begin{tabular}{|c|c|c|c|c|c|}
\hline Variável & $\begin{array}{l}\text { Média } \\
\text { (dias) }\end{array}$ & $\begin{array}{l}\text { Mediana } \\
\text { (dias) }\end{array}$ & $\begin{array}{l}\text { Desvio-padrão } \\
\text { (dias) }\end{array}$ & $\begin{array}{l}\text { Mínimo } \\
\text { (dias) }\end{array}$ & $\begin{array}{l}\text { Máximo } \\
\text { (dias) }\end{array}$ \\
\hline Primeira internação & 17,3 & 12,0 & 16,7 & 1 & 99 \\
\hline $\begin{array}{c}\text { Tempo entre a primeira internação e primeira } \\
\text { readmissão }\end{array}$ & 51,6 & 23,5 & 70,4 & 1 & 265 \\
\hline Primeira readmissão & 16,9 & 13,0 & 14,5 & 1 & 62 \\
\hline $\begin{array}{l}\text { Tempo entre a primeira e segunda } \\
\text { readmissão }\end{array}$ & 58,6 & 14,5 & 82,4 & 7 & 271 \\
\hline Segunda readmissão & 20,6 & 13,5 & 26,7 & 1 & 90 \\
\hline $\begin{array}{l}\text { Tempo entre a segunda e a terceira } \\
\text { readmissão }\end{array}$ & 5,0 & 5,0 & 5 & 5 & 5 \\
\hline Terceira readmissão & 11,0 & 11,0 & 0 & 11 & 11 \\
\hline
\end{tabular}

Entre os pacientes que desenvolveram ISC ( $n=24), 19(79,2 \%)$ foram readmitidos entre oito e 30 dias, três $(12,5 \%)$ em sete dias e dois (8,3\%) em um período superior a 30 dias.

Na Tabela 3, observam-se as características sociodemográficas dos pacientes readmitidos após cirúrgica cardíaca e sua relação com a principal complicação na primeira readmissão, ou seja, ISC.

Observa-se que os pacientes readmitidos eram predominantemente homens, acima dos 60 anos, hipertensos e dislipidêmicos. Houve associação estaticamente significante entre a variável readmissão por ISC e as variáveis dislipidemia $(p=0,007)$ e obesidade $(p=0,05)$ (Tabela 3).

Cabe destacar que, entre os casos que apresentaram ISC, 21 (87,5\%) pacientes haviam sido submetidos à RM e apenas três $(12,5 \%)$ à IPV $(p<0,001)$. A análise de regressão logística indicou que os indivíduos que realizaram RM na primeira internação tiveram 16,7 vezes mais chances de serem readmitidos por ISC, quando comparados àqueles que realizaram implante de prótese valvar. 
Tabela 3: Distribuição das variáveis de caracterização dos pacientes submetidos à revascularização do miocárdio e implante de prótese valvar e readmissão por infecção do sítio cirúrgico. São Paulo, SP, Brasil, 2012.

\begin{tabular}{|c|c|c|c|c|c|}
\hline \multirow{2}{*}{ Variáveis } & \multicolumn{2}{|c|}{ Readmissão } & \multicolumn{3}{|c|}{ Readmissão por ISC } \\
\hline & $N(62)$ & $\%$ & $N(24)$ & $\%$ & $\bar{p}$ \\
\hline Sexo & & & & & $0,780^{*}$ \\
\hline Masculino & 41 & 66,1 & 15 & 62,5 & \\
\hline Feminino & 21 & 33,9 & 9 & 37,5 & \\
\hline Faixa Etária & & & & & $0,390^{+}$ \\
\hline $18-40$ anos & 6 & 9,7 & 0 & 0 & \\
\hline $41-60$ anos & 25 & 40,3 & 11 & 45,8 & \\
\hline 61 anos ou mais & 31 & 50,0 & 13 & 54,2 & \\
\hline Estado civil & & & & & $0,640 *$ \\
\hline Solteiro & 7 & 11,4 & 2 & 8,3 & \\
\hline Casado & 42 & 67,7 & 15 & 62,5 & \\
\hline Viúvo & 3 & 4,8 & 2 & 8,3 & \\
\hline Separado/divorciado & 8 & 12,9 & 4 & 16,6 & \\
\hline Não preenchido & 2 & 3,2 & 1 & 4,2 & \\
\hline Escolaridade & & & & & $0,950 *$ \\
\hline Analfabeto & 6 & 9,8 & 2 & 8,3 & \\
\hline 1-11 anos de estudo & 43 & 69,3 & 16 & 66,7 & \\
\hline 12 anos ou mais de estudo & 10 & 16,1 & 4 & 16,7 & \\
\hline Não preenchidos & 3 & 4,8 & 2 & 8,3 & \\
\hline \multicolumn{6}{|l|}{ Doenças crônicas } \\
\hline Hipertensão Arterial Sistêmica & 49 & 79,0 & 20 & 83,3 & $0,500 *$ \\
\hline Dislipidemia & 28 & 45,2 & 16 & 66,7 & $0,007 *$ \\
\hline Diabetes mellitus & 18 & 29,0 & 9 & 37,5 & $0,250 *$ \\
\hline Obesidade & 13 & 21,0 & 8 & 33,3 & $0,050 *$ \\
\hline Tabagismo & 6 & 9,7 & 2 & 8,3 & $1,000 *$ \\
\hline Situação trabalhista & & & & & $0,860^{*}$ \\
\hline Atividade remunerada & 29 & 46,8 & 9 & 37,5 & \\
\hline Aposentado & 16 & 25,8 & 0 & 0 & \\
\hline Afastado & 3 & 4,8 & 0 & 0 & \\
\hline Do Lar & 10 & 16,1 & 4 & 16,7 & \\
\hline Outros & 4 & 6,5 & 11 & 45,8 & \\
\hline Procedência & & & & & $0,470^{*}$ \\
\hline São Paulo - capital & 32 & 51,6 & 14 & 58,3 & \\
\hline São Paulo - interior & 27 & 43,6 & 10 & 41,7 & \\
\hline Outros estados & 3 & 4,8 & 0 & 0 & \\
\hline
\end{tabular}

${ }^{*}$ Teste exato de Fisher; ${ }^{+}$Teste t de Student.

\section{DISCUSSÃO}

Os dados encontrados na atual investigação estabelecem relação com a literatura científica em relação a principal causa de readmissão, a ISC, e a associação das comorbidades como fatores significativos para sua ocorrência ${ }^{(9,12)}$, no entanto, predominaram homens na amostra investigada e taxas de readmissão inferiores as evidenciadas pela literatura científica ${ }^{(9,13)}$.

Estudo americano analisando 11.823 altas hospitalares, entre pacientes submetidos à RM, observou uma taxa de readmissão, em 30 dias, de 13,2\%, sendo as infecções pós-operatórias as causas mais frequentes de readmissão. Houve associação entre a readmissão e características dos pacientes como, serem idosos, do sexo feminino e apresentarem comorbidades pré-operatórias ${ }^{(9)}$.

Outra investigação analisando 180.568 pacientes submetidos a IPV, entre 1999 e 2010, verificou que 
49,1\% destes, principalmente mulheres, idosas e negras foram readmitidas em um ano após a cirurgia por falência cardíaca, arritmias, sepse e complicações pós-operatórias, com duração média de hospitalização de 38 dias após a readmissão ${ }^{(12)}$.

Na presente investigação, os pacientes levaram, em média, 52 dias para serem readmitidos e metade deles permaneceu até 13 dias hospitalizados durante a readmissão, valores contrastantes aos encontrados em estudo anterior, que também analisou cirurgias de RM e IPV e encontrou taxas de readmissão em 30 dias de $13 \%$, com mediana de readmissão em seis dias após a alta e nova internação com duração entre um e 138 $\operatorname{dias}^{(13)}$.

A principal causa de readmissão neste estudo foi a ISC; sabe-se que seu desenvolvimento está vinculado a maior morbidade e complicações a longo prazo, aumentando o tempo de estadia hospitalar e custos $^{(14)}$. Espera-se que a incidência de ISC em RM e IPV, por se tratarem de cirurgias consideradas limpas, variem entre 0,2 e $5,6^{(15-16)}$.

Dessa forma, vários fatores podem influenciar o aparecimento de infecções graves pós-operatórias, entre eles, a obesidade, cirurgia cardíaca prévia ou de emergência, insuficiência renal, imunossupressão, falência cardíaca e doenças cerebrovasculares ou periféricas ${ }^{(17)}$.

O tabagismo é um preditor para complicações pós-operatórias por causar deterioração da função pulmonar ${ }^{(18)}$ e celulares inflamatórias e reparadoras, o que ocasiona atraso na cicatrização, necrose, ISC e deiscência, por exemplo. Logo, considera-se que a cessação do fumo por até quatro semanas, ou 30 dias, antes do procedimento cirúrgico seja capaz de recuperar as funções celulares, embora, a resposta proliferativa celular permaneça prejudicada ${ }^{(19)}$.

Outro aspecto a ser evidenciado é a associação entre obesidade e dislipidemia, que torna os pacientes mais susceptíveis ao desenvolvimento de aterosclerose coronariana, que tem como desfecho frequente a cirurgia de RM. Ressalta-se que, em muitos indivíduos, o comprometimento é tão extenso que a terapêutica medicamentosa ou percutânea é insuficiente para manter uma boa qualidade de vida ${ }^{(20-21)}$.

Pacientes obesos submetidos à cirurgia cardíaca têm maior frequência de complicações pósoperatórias importantes, como ISC, tromboembolia pulmonar e síndrome da resposta inflamatória sistêmica ${ }^{(20-21)}$. A obesidade é um fator de risco independente para a mediastinite, além de estar associada ao aumento da estadia hospitalar, menor sobrevida em cinco anos e dificuldade de distribuição sistêmica da antibioticoprofilaxia $^{(20)}$.

Outro importante fator de risco tanto para doença cardiovascular (DCV), quanto para ISC é o diabetes mellitus (DM). A associação entre DM e DCV está bem estabelecida, indicando que a presença isolada de DM aumenta o risco de doença arteriovascular e acidente vascular encefálico de duas a quatro vezes, quando comparado com indivíduos não diabéticos ${ }^{(22)}$. Estudo nacional demonstrou que $45,7 \%$ dos pacientes que foram readmitidos por mediastinite eram diabéticos ${ }^{(23)}$.

A hiperglicemia estimula a liberação de certos mediadores inflamatórios, dificultando a cicatrização e comprometendo a imunidade, o que levaria ao prejuízo no combate de infecções ${ }^{(23)}$, devendo ser objetivo 
da equipe de saúde a manutenção da glicemia sanguínea do indivíduo em níveis inferiores a $200 \mathrm{mg} / \mathrm{dl}$ nos primeiros dois dias pós-operatórios ${ }^{(20,23)}$.

Neste contexto, o paciente submetido a uma RM, na qual não houve complicações, permanecerá em média sete dias hospitalizado, com período previsto de recuperação entre seis e 12 semanas $^{(4)}$. Portanto, o desenvolvimento de ISC em cirurgia cardíaca merece atenção, pois prolonga o tempo de internação do paciente, o expõe a elevada morbimortalidade, causa importante impacto econômico e social, elevando os custos da internação ${ }^{(14-16)}$ e refletindo em possível readmissão ${ }^{(6,8-9)}$.

Destaca-se também, a repercussão que a cirurgia cardíaca traz a vida do paciente a ela submetido, com desafios em relação à manutenção do estilo de vida e a necessidade de suporte por parte da equipe de saúde ${ }^{(24)}$. Deste modo, cabe a equipe de saúde estabelecer programas que minimizem possíveis complicações pós-operatórias, como a ISC, que impactarão de forma negativa no sucesso do tratamento.

\section{CONCLUSÃO}

Conclui-se que a maior parte dos pacientes readmitidos após a realização de cirurgia cardíaca eram homens, com mais de 60 anos, hipertensos, sedentários e dislipidêmicos, submetidos à revascularização do miocárdio. A taxa de readmissão após RM e IPV foi de 5,9\%, inferior à observada na literatura científica.

A principal causa de readmissão do paciente cirúrgico foi a infecção no sítio cirúrgico. As comorbidades obesidade e dislipidemia estiveram associadas de forma estatisticamente significante a infecção no sítio cirúrgico.

Diante destes achados, reforça-se a necessidade de um planejamento de manejo perioperatório multiprofissional para as cirurgias cardíacas eletivas, por meio da idealização de ações de controle dos fatores de risco para o desenvolvimento de infecção do sítio cirúrgico, que foi, na instituição analisada, a principal causa de readmissão.

Neste sentido, novas investigações devem ser conduzidas, em especial, por enfermeiros, que são os responsáveis pelo cuidado perioperatório destes pacientes, buscando estabelecer quais as melhores proposições para o controle dos fatores intrínsecos associados ao paciente, que contribuem para o aumento dos casos de ISC e posterior readmissão.

Como limitação do estudo é importante ressaltar a coleta retrospectiva dos dados, que enfrenta problemas relativos à qualidade de registros, mas que permite a análise de um maior conjunto de procedimentos.

\section{REFERÊNCIAS}

1. Duncan BB, Chor D, Aquino EML, Bensenor IM, Mill JG, Schmidt MI, et. al. Chronic Non-communicable diseases in Brazil: priorities for disease management and research. Rev Saúde Publica [Internet]. 2012 [citado 7 jun.

2016];46(Suppl):126-34. Disponível em: http://www.scielo.br/pdf/rsp/v46s1/17.pdf.

2. Mansur AP, Favarato D. Mortalidade por doenças cardiovasculares no Brasil e na região metropolitana de São Paulo: Atualização 2011. Arq Bras Cardiol. 2012;99(2):755-61.

3. Pereira KSM, Oliveira JCP, Carvalho FC, van Bellen B. Complicações cardíacas em cirurgia vascular Cardiac 
complications in vascular surgery. J Vasc Bras [Internet]. 2016 [citado 7 jun. 2016];15(1):16-20. Disponível em: http://jvascbras.com.br/pdf/16-15-01/03 jvbAO20150035 PT.pdf.

4. Alexander JH, Smith PK. Coronary-Artery Bypass Grafting. N Engl J Med. 2016;374(20):1954-64.

5. Nishimura RA, Otto CM, Bonow RO, Carabello BA, Erwin JP, Guyton RA, et al. 2014 AHA/ACC Guideline for the Management of Patients With Valvular Heart Disease: Executive Summary: A Report of the American College of Cardiology/American Heart Association Task Force on Practice Guidelines. Circulation [Internet]. 2014 [acesso em: 31 mar. 2016];129(23):2440-92. Disponível em: http://dx.doi.org/10.1161/CIR.0000000000000029.

6. Laizo A, Delgado FE, Rocha GM. Complications that increase the time of Hospitalization at ICU of patients submitted to cardiac surgery. Rev Bras Cir Cardiovasc. 2010; 25(2):166-71.

7. Tsai TC, Joynt KE, Orav J, Gawande AA, Jha AK. Variation in surgical-readmission rates and quality of hospital care. $\mathrm{N}$ Engl J Med. 2013;369(12):1134-42. DOI: 10.1056/NEJMsa1303118.

8. Lancey R, Kurlansky P, Argenziano M, Coady M, Dunton R, Greelish J, et al. Uniform standards do not apply to readmission following coronary artery bypass surgery: a multi-institutional study. J Thorac Cardiovasc

Surg. 2015;149(3):850-7. DOI: 10.1016/j.jtcvs.2014.08.059.

9. Li Z, Armstrong EJ, Parker JP, Danielsen B, Romano PS. Hospital variation in readmission after coronary artery bypass surgery in California. Circ Cardiovasc Qual Outcomes. 2012;5(5):729-37.

10. Chen JC, Shaw JD, Ma Y, Rhoads KF. The role of the hospital and health care system characteristics in readmissions after major surgery in California. Surgery. 2016;159(2):381-8. DOI: 10.1016/j.surg.2015.06.016.

11. Resolução № 466 do Conselho Nacional de Saúde, de 12 de dezembro de 2012 (BR). Aprova as diretrizes e normas regulamentadoras de pesquisas envolvendo seres humanos. Diário Oficial da União. 12 dez 2012.

12. Dodson JA, Wang Y, Murugiah K, Dharmarajan K, Cooper Z, Hashim S, et al. National Trends in Hospital Readmission Rates among Medicare Fee-for-Service Survivors of Mitral Valve Surgery, 1999-2010. PLoS ONE. 2015;10(7):e0132470. Disponivel em: http://dx.doi.org/10.1371/journal.pone.0132470.

13. Price JD, Romeiser JL, Gnerre JM, Shroyer AL, Rosengart TK. Risk analysis for readmission after coronary artery bypass surgery: developing a strategy to reduce readmissions. J Am Coll Surg. 2013;216(3):412-9.

14. Awad SS. Adherence to Surgical Care Improvement Project Measures and Post-Operative Surgical Site Infections. Surg Infect. 2012;13(4):234-237.

15. Silva QCG, Barbosa MH. Risk factors for surgical site infection in cardiac surgery. Acta Paul Enferm. 2012;25(2):8995.

16. Mangram AL; Horan TC; Pearson ML; Silver LC; Jarvis WR, et al. Guideline for prevention of surgical site infection, 1999. Infect Control and Hosp Epidemiol.1999;20(4):247-78.

17. Chen LF, Arduino JM, Sheng S, Muhlbaier LH, Kanafani ZA, Harris AD, et al. Epidemiology and outcome of major postoperative infections following cardiac surgery: risk factors and impact of pathogen type. Am J Infect Control. 2012;40(10):963-8.

18. Oliveira JC, Fantinati MS. Perfil de pacientes submetidos a cirurgia cardíaca e preditores das complicações pósoperatórias. Revista biomotriz. 2013;7(1):64-78.

19. Sørensen LT. Wound healing and infection in surgery: the pathophysiological impact of smoking cessation, and nicotine replacement therapy: a systematic review. Ann Surg. 2012;255(6):1069-79.

20. Lepelletier D, Bourigault $C$, Roussel JC, Lasserre $C$, Leclère $B$, Corvec $S$, et al. Epidemiology and prevention of surgical site infections after cardiac surgery. Med Mal Infect. 2013;43(10):403-9.

21. Araujo NR, Araújo RA, Bezerra SMMS. Overweight And Obesity Repercussion In The Postoperative Of Myocardial Revascularization Surgery. Rev Esc Enferm USP. 2014;48(2):236-41.

22. Santos PA, Pinho CPS. Diabetes mellitus pacientes coronariopatas: prevalência e fatores de risco cardiovascular associado. Rev Bras Clin Med. 2012;10(6):469-75.

23. Tiveron MG, Fiorelli Al, Mota E.M, Vilca OM, Brandão CMA, et al. Preoperative risk factors for mediastinitis after cardiac surgery: assessment of 2768 patients. Rev Bras Cir Cardiovasc. 2012;27(2):203-10.

24. Erdmann AL, Lanzoni GMM, Callegaro GD, Baggio MA, Koerich C. Understanding the process of living as signified by myocardial revascularization surgery patients. Rev Lat Am Enfermagem [Internet]. 2013 Fev [acesso em: 08 dez. 2016];21(1):332-9. Disponível em: http://dx.doi.org/10.1590/S0104-11692013000100007. 